\title{
Stability of an Acetylene Frequency Reference inside Kagome Structured Hollow-Core Photonic Crystal Fiber
}

\author{
Kevin Knabe ${ }^{1}$, Jinkang Lim ${ }^{1}$, Karl Tillman ${ }^{1}$, Rajesh Thapa ${ }^{1}$, François Couny ${ }^{2}$, Phillip S. Light ${ }^{2}$, \\ Jeffrey W. Nicholson ${ }^{3}$, Brian R. Washburn ${ }^{1}$, Fetah Benabid ${ }^{2}$, and Kristan L. Corwin ${ }^{1}$ \\ ${ }^{1} 116$ Cardwell Hall, Department of Physics, Kansas State University, Manhattan, KS 66506, U.S.A \\ ${ }^{2}$ Centre for Photonics and Photonics Materials, Dept. of Physics, University of Bath, BA2, 7AY, UK \\ ${ }^{3}$ OFS Labs, Somerset, NJ 08873 USA \\ kok6785@ksu.edu,corwin@phys.ksu.edu
}

\begin{abstract}
A continuous-wave laser has been stabilized to an acetylene transition inside kagome photonic crystal fiber. Stability as measured with a carbon nanotube fiber laser frequency comb to is better than $1 \times 10^{-11}$ at $10 \mathrm{~s}$. (C)2009 Optical Society of America

OCIS codes: 120.3930 (Metrological instrumentation), (320.7090) Ultrafast lasers; (060.5295) Photonic crystal fibers
\end{abstract}

\section{Introduction}

Wavelength references in the near IR are important for the telecommunications industry, length metrology, and other applications. Acetylene wavelength references based on power build-up cavities and vapor cells have achieved accuracies of $\sim 2 \mathrm{kHz}$ and stabilities of $\sim 3 \times 10^{-12}$ at $1 \mathrm{~s}$ with sub-Doppler linewidths of $\sim 1 \mathrm{MHz}$ [1-3]. Hollow core optical fiber offers a robust system in which to perform nonlinear optics in gases [4]. Saturated absorption in hollow-core photonic crystal fiber (HC-PCF) offers the potential advantage of all-fiber portability; however, the smaller beam size results in larger linewidths, making the high accuracy and stability of the power build-up cavity based references difficult to achieve. In $10 \mu \mathrm{m} \mathrm{HC}-\mathrm{PCF}, 34 \mathrm{MHz}$ (15 MHz) FWHM linewidths at high (low) pump powers have been achieved. Signal-to-noise ratios are high enough that stability of $10^{-11}$ in $1 \mathrm{~s}$ should be possible [5], which is comparable with $\mathrm{I}_{2}$ - stabilized HeNe lasers. Kagome structured HC-PCF offers large core sizes $(\sim 70 \mu \mathrm{m})$ with moderate loss $(\sim 1 \mathrm{~dB} / \mathrm{m})$ resulting in transit time limited sub-Doppler features of $\sim 10$ $\mathrm{MHz}$ linewidths, and higher signal-to-noise ratios. Here we demonstrate the stability of such a reference.

\section{Setup for Frequency Stabilization of a cw Laser}

A continuous-wave (CW) fiber laser was locked to a kagome-structured HC-PCF acetylene reference using the optical and electrical set-ups shown in Figs. 1a-b [6]. A narrow linewidth CW fiber laser (Orbits Lightwave, 25 $\mathrm{mW}, 1532 \mathrm{~nm}$ ) is split using a fiber coupler to create the pump beam (Fig. 1a). This pump light passes through an amplitude modulator (AM) driven at $f_{A M}=900 \mathrm{kHz}$. An erbium doped fiber amplifier (EDFA) increases the pump power up to $250 \mathrm{~mW}$. The pump beam is coupled to free space, and then into a $1.4 \mathrm{~m}$ large-core (70 micron) kagome-structured HC-PCF whose ends reside inside vacuum chambers filled with acetylene to 100 mtorr. The remaining $\mathrm{CW}$ light from the fiber laser becomes the probe beam and an additional beam to beat against a frequency comb to characterize stability and accuracy. The probe beam passes through an acousto-optic modulator (AOM) to shift any interference between probe and stray pump reflections to the AOM frequency. The probe next passes through an electro-optic modulator (EOM), creating sidebands spaced at $f_{F M}=22 \mathrm{MHz}$ for FM spectroscopy [7]. The probe beam is coupled to free space and then into the kagome HC-PCF, but anti-parallel to the pump beam.

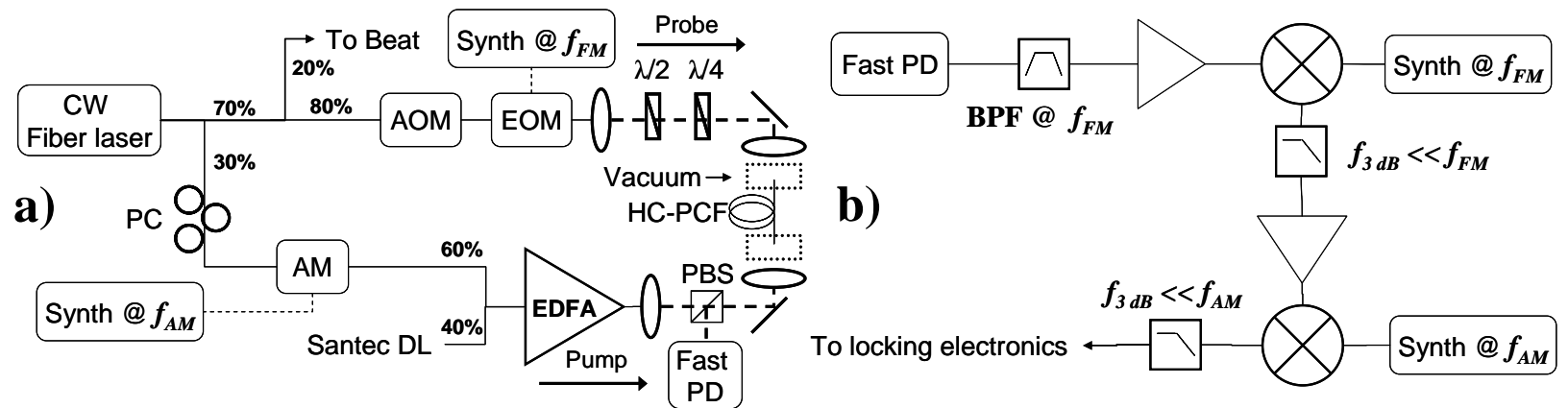

Fig. 1: a) Optical schematic for saturated absorption spectroscopy including frequency modulation of probe and amplitude modulation of pump. A Santec TSL-210 diode laser (DL) is tuned off-resonance and coupled into the EDFA with the chopped fiber laser so that the amplifier always sees an input $\left(\lambda_{\mathrm{DL}}=1548 \mathrm{~nm}\right)$. Synth: Synthesizer, DL: diode laser, and PC: polarization controller. Numbers at intersections indicate coupling percentages in fiber couplers. b) Electrical schematic used to retrieve the background-free derivative-like error signal. 
Polarization optics are used to separate these two beams at the polarizing beam splitter (PBS) in the free space section of the pump beam, and the resulting probe light is then detected with a $125 \mathrm{MHz}$ New Focus photodetector (PD).

Figure $1 \mathrm{~b}$ details the electrical setup used to extract the error signal for stabilizing the $\mathrm{CW}$ laser to an acetylene transition. The RF signal from the PD is filtered, amplified, and then mixed with $f_{F M}$ to extract a derivative-like signal of the sub-Doppler feature. The output of this mixer is then low-pass filtered (LPF) well below $f_{F M}$ at $60 \mathrm{kHz}$, amplified, and mixed with $f_{A M}$. The subsequent LPF then produces a lock-in type signal, where background offsets due to anything except the presence of the pump beam are eliminated. This signal, shown in Fig 2a to be on the order of $10 \mathrm{MHz}$ peak-to-peak, is the error signal used to feed back to the fiber laser. The optimum relative stability of this reference was calculated to be $6 \times 10^{-12} \mathrm{~Hz}^{-1 / 2}$ by comparing the off-resonant noise $(10 \mathrm{mV})$ to the slope of the feature $(30 \mathrm{MHz} / \mathrm{V})$ within the $60 \mathrm{kHz}$ bandwidth and assuming white noise.

\section{Stability of the acetylene stabilized cw laser}

A portion of the probe light is split before the AOM and EOM and used to beat with a phase-stabilized carbon nanotube fiber laser (CNFL) frequency comb $[8,9]$. The resulting RF frequency was counted with a HP53131A frequency counter at either 0.1 or $1 \mathrm{~s}$ gate times and fractional Allan deviations were recorded. Higher averaging times were obtained by averaging this data. The stability reported here is within a factor of 20 of the previous nonfiber-based references. Previous measurements of acetylene gas cells offer better than a factor ten improvement on our measurements, perhaps due to the narrower sub-Doppler linewidth. Measurements of the absolute frequency of this reference has been measured with a Cr:forsterite laser comb by changing its repetition rate [10] and are reproducible to $<200 \mathrm{kHz}$. Some shifts due to coupling and fiber position have been observed, which may be related to the multi-mode nature of the fiber. Further investigation is ongoing to improve both stability and repeatability, and to determine the fundamental limits inside large-core kagome-structured HC-PCF.
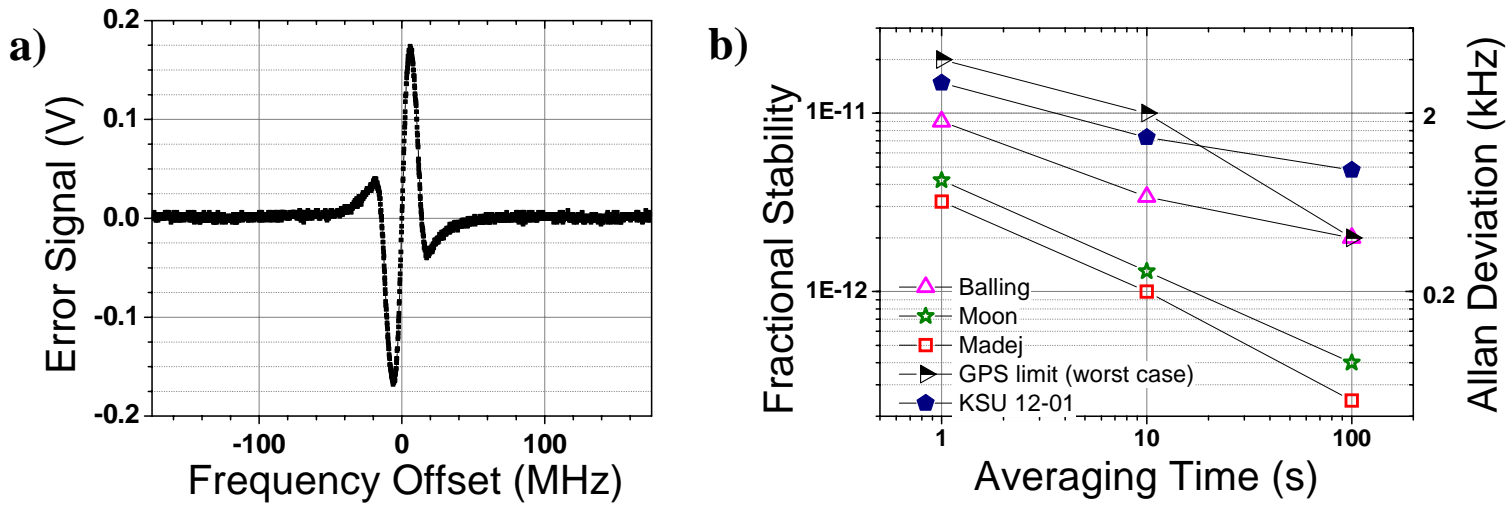

Fig. 2a) Amplitude of error signal versus frequency offset around the $\mathrm{P}(13) v_{1}+v_{3}$ transition in acetylene. b) Fractional stability of the beat between the frequency stabilized CW fiber laser and the phase-stabilized carbon nanotube frequency comb (KSU) as compared with other optical references based on acetylene [1-3]. The CNFL comb is stabilized to a GPS-disciplined Rb clock, and is limited by it.

\section{References}

[1] P. Balling, M. Fischer, P. Kubina, and R. Holzwarth, "Absolute frequency Absolute frequency measurement of wavelength standard at 1542nm: acetylene stabilized DFB laser," Opt. Exp. 13, 9196-9201 (2005).

[2] A.A. Madej, J.E. Bernard, A.J. Alcock, A. Czajkowski, and S. Chepurov, "Accurate absolute frequencies of the $v_{1}+v_{3}$ band of ${ }^{13} \mathrm{C}_{2} \mathrm{H}_{2}$ determined using an infrared mode-locked Cr:YAG laser frequency comb," J. Opt. Soc. Am. B 23, 741-749 (2006).

[3] H.S. Moon, W.K. Lee, and H.S. Suh, "Absolute-Frequency Measurement of an Acetylene-Stabilized Laser Locked to the P(16) Transition of ${ }^{13} \mathrm{C}_{2} \mathrm{H}_{2}$ Using an Optical-Frequency Comb," in IEEE Trans. on Intrs. and Meas. 56, 509-512 (2007).

[4] F. Benabid, "Hollow-core photonic bandgap fibres: new light guidance for new science and technology," Phil. Tran. R. Soc. A 364, 3439$3462(2006)$.

[5] J.C. Petersen and J. Hald, "Frequency and Wavelength Standards Based on Gas Filled HC-PBFs," in Proceedings of Conference on Lasers and Electro-optics (CLEO) JFA1, (Optical Society of America, 2008).

[6] K. Knabe, A. Jones, K. L. Corwin, F. Couny, P. S. Light, and F. Benabid, "Saturated absorption spectroscopy of $\mathrm{C}_{2} \mathrm{H}_{2}$ inside hollow, largecore kagome photonic crystal fiber," in Proceedings of CLEO JFA5, (Optical Society of America, 2008).

[7] J. L. Hall, L. Hollberg, T. Baer, and H. G. Robinson, “Optical heterodyne saturation spectroscopy,” Appl. Phys. Lett. 39, 680-682 (1981).

[8] J. Nicholson and D. J. DiGiovanni, "High repetition frequency, low noise, fiber ring lasers modelocked with carbon nanotubes," IEEE Photonics Tech. Lett. 20 (2008).

[9] Y. Wang, J. Lim, R. Amezcua-Correa, J. C. Knight, and B. R. Washburn, "Sub-33 fs Pulses from an All-Fiber Parabolic Amplifier Employing Hollow-Core Photonic Bandgap Fiber," in Proceedings of Frontiers in Optics, (Optical Society of America, 2008).

[10] L.S. Ma, M. Zucco, S. Picard, L. Robertsson, and R.S. Windeler, “A New Method to Determine the Absolute Mode Number of a ModeLocked Femtosecond-Laser Comb Used for Absolute Optical Frequency Measurements," IEEE J. Quantum Electron. 9, 1066-1071 (2003). 